\title{
Incorporating firebrands and spot fires into vorticity-driven wildfire behaviour models
}

\author{
$\underline{\text { J.E. Hilton }}^{\mathrm{a}}$, N. Garg ${ }^{\mathrm{a}}$ and J.J. Sharples ${ }^{\mathrm{b}}$

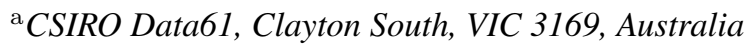 \\ ${ }^{\mathrm{b}}$ School of Science, UNSW Canberra, ACT 2600, Australia \\ Email: james.hilton@data61.csiro.au
}

\begin{abstract}
Complex modes of fire behaviour resulting from local coupling between the fire and the atmosphere are a significant challenge for rapid operational wildfire spread simulations. While threedimensional fully coupled fire-atmosphere models are able to account for many types of fire behaviour, their computational demands are prohibitive in an operational context. Two-dimensional fire spread models have much lower computational overhead, but are generally not able to account for complex local coupling effects and cannot provide a three-dimensional flow structure suitable for modelling the transport of firebrands. In this paper we investigate extending two-dimensional fire spread simulations to model local coupling effects resulting from wind flow over a ridge that can result in a number of non-intuitive modes of fire behaviour. These include fire propagation opposite to the direction of the prevailing wind on the lee slope of ridges caused by re-circulation on the lee slope, called vorticity-driven lateral spread (VLS). Furthermore we develop extensions of these two-dimensional models to incorporate three-dimensional firebrand transport and show that enhanced downwind spot fire formation can result under certain VLS conditions.

The spread of fires under VLS conditions is driven by vortices in the ground plane. A model for the production and effects of these vortices was incorporated into computational simulations using a vector potential formu-lation in similar manner to a scalar 'pyrogenic potential' model, detailed in earlier studies. Firebrands were incorporated using a Lagrangian scheme to model transport through the atmosphere and a sub-scale model for spot fire creation and growth. The firebrand transport took factors such as drag, gravity and buoyancy into account. As effect of plume buoyancy on firebrands under real-world conditions for this scenario is currently unknown, the plume buoyancy was parameterised using a exponential decay model. The sensitivity of the decay parameter in this model was then examined in relation to the resulting spot fire distribution and area burnt. All simulations were carried out using Spark, a wildfire prediction framework.

The coupled VLS and firebrand transport simulations indicated that a higher value of decay parameter, rep-resenting a higher cooling rate of the plume, acted to enhance the lateral spread as firebrands were lofted for shorter times and were caught in the vortices at the edge of the lateral spread region. In contrast, a lower value of decay parameter, representing a lower cooling of the plume, resulted in widespread downwind spot fires and larger burnt areas. This appeared to be due to longer lofting times resulting in firebrands being transported further downwind and away from the vortices within the lateral spread region. The model appears, at least qualitatively, to match observed lateral spread and 'deep flaming' fire behaviour although many of the parame-ters in the model require further research and experimental calibration. Further development of the model may allow these complex modes of fire behaviour to be incorporated into rapid wildfire models for operational and risk assessment usage.
\end{abstract}

Keywords: Firebrands, embers, spotting, wildfire simulation, dynamic fire propagation 


\section{INTRODUCTION}

Fire spread simulators play an essential part in wildfire risk assessment and management, but their effectiveness is limited in a number of ways. In particular, their performance is critically dependent on the accuracy of the input information on weather, fuels and terrain and the ability of the underpinning fire spread models and propagation algorithms to faithfully represent the main processes driving fire propagation. This second dependence becomes critical when the spread of a wildfire is dominated by the dynamic modes of fire propagation, which arise in response to multi-scale interactions between the fire and the local atmosphere. Examples of these dynamic modes of fire propagation include eruptive fire spread (Viegas, 2006; Viegas and Pita, 2004) and vorticity-driven lateral spread (Sharples et al., 2012; Simpson et al., 2013, 2014, 2016).

The VLS phenomenon, in particular, has been shown to be critical in the development of extreme wildfires (McRae et al. 2015). VLS is characterised by rapid lateral propagation of a fire across the top of lee-facing slopes, but its influence on extreme wildfire development can be mostly attributed to the secondary generation of firebrands, massive ember attack and spot fire coalescence downwind of the lateral spread zones. The strong vorticity associated with the lateral spread enhances the production of embers, which are lofted into the plume and transported downwind by the prevailing winds. Hence, as the fire spreads laterally, it 'casts off' embers downwind, which then produce large areal flaming zones - referred to as a 'deep flaming' event.

Figure 1 shows an example of how VLS produces deep flaming. The fire depicted in Fig. 1 is the Yankees Gap fire, which burnt on the $15^{\text {th }}$ of September 2018 on the south coast of NSW. The Fig. 1 shows that a small fire ignited close to 13:20 hours (see Fig. 1'a)), burns against the northwesterly winds back up a leeward slope until it hits the ridge line. The fire then spreads laterally across the wind towards the south west (consistent with VLS) and 'spills out' downwind as a mass of spot fires, which coalesce into the large (approx. 400 ha) flaming zone visible in Fig. 1'b).
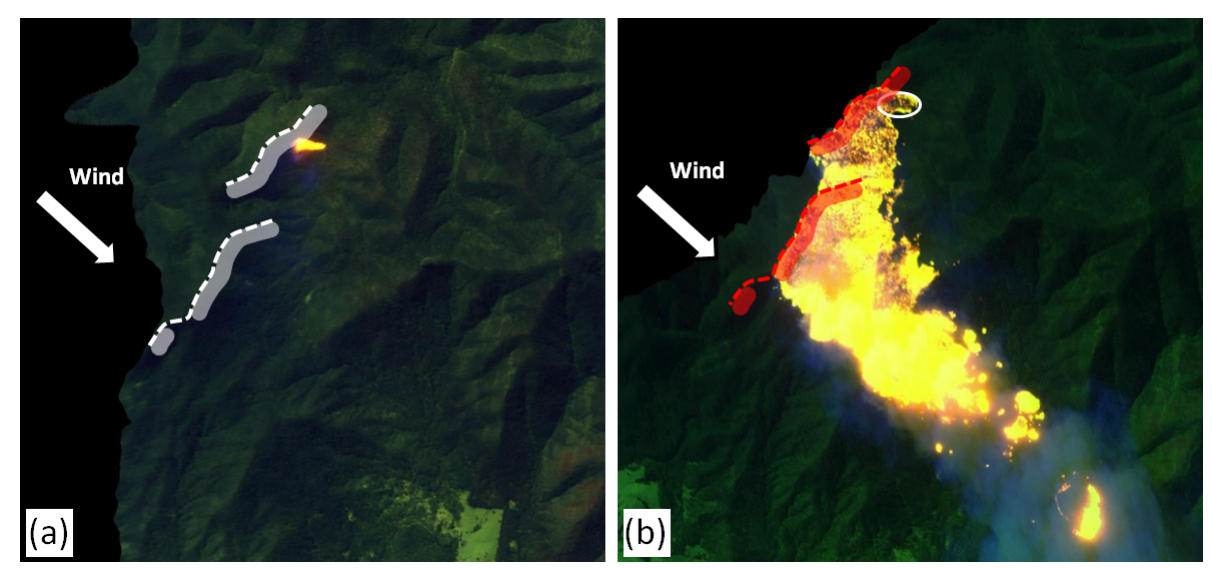

Figure 1. Yankees Gap fire NSW, $15^{\text {th }}$ September 2018. (a) a small fire ignited at 13:20 hours burns back against the prevailing wind direction (indicated by the white arrow). The white dashed lines indicate ridge lines, while the grey shading indicates regions prone to VLS. (b) The fire approximately 50 minutes later at 14:17 hours. The white ellipse shows the location of the small fire from panel (a), while the red dashed lines and shading are the same as the white ones in panel (a). Linescan images were provided by NSW Rural Fire Service.

Current operational fire spread simulators (e.g. Finney (2004); Tolhurst et al. (2008)) are poorly suited for modelling dynamic fire propagation. This is mainly due to their reliance on the assumption that a fire will spread at a quasi-steady rate uniquely determined by the environmental conditions, and the assumption that different parts of a fire line propagate independently. This latter assumption, for example, is implicit in propagation algorithms such as those based on Huygens' principle, which is often used in operational fire spread simulators. Given the dramatic effect that dynamic processes such as VLS can have on the development of a fire, the inability to properly account for them in operational fire spread simulators constitutes a significant gap in operational capability. The issue, however, is that dynamic modes of fire propagation are driven by complex interactions between the fire and the atmosphere, or between different parts of the fire itself. Incorporating such effects in models that are able to run within operational time frames is a significant challenge. 
At present it is only possible to accurately model phenomena like VLS using three-dimensional computational fluid dynamics models or fully coupled fire-atmosphere models. The computational cost associated with these modelling approaches makes them in-feasible for operational use - simulations of even fairly modest sized fires can take the order of 10-20 hours on a supercomputer, whereas operational needs require simulations that run much faster than real time. Recently, Hilton et al. (2018) introduced a model that permits inclusion of dynamic interactions between different parts of a fire, thereby relaxing the assumptions that rate of spread is quasi-steady and that different parts of a fire burn independently. Although this approach is still manifestly two-dimensional, it has been used to successfully model a number of different modes of dynamic fire spread such as the behaviour of junction fires. The two-dimensional nature of the model means that it is able to run much faster than real time, yet is still able to reproduce fire spread features that have previously required fully coupled fire-atmosphere models to resolve.

Even more recently, Sharples and Hilton (2019) demonstrated how the inclusion of vorticity effects as part of a two-dimensional near-field modelling approach could be used to emulate the pattern of fire propagation associated with VLS. In particular, they showed that output from the two-dimensional model compared favourably with that obtained from a fully coupled fire-atmosphere model. However, at present this modelling only captured the lateral spread associated with VLS and no attempt was made to model the spotting component that produces deep flaming. Therefore, in this paper we combine the model considered by Sharples and Hilton (2019) with a Lagrangian firebrand model to simulate the transport of firebrands and subsequent spot fire formation.

We begin by giving a brief recount of VLS before explaining the two-dimensional modelling approach and incorporating the spotting component. The utility of the combined model is thereafter illustrated with an idealised example.

\section{VORTICITY-DRIVEN LATERAL SPREAD}

The presence of atypical modes of fire propagation under certain conditions was first reported by McRae (2004) from examination of line-scan data from the 2003 Canberra wildfires. This behaviour was characterised by rapid lateral fire spread across the top of a steep leeward slope in a direction approximately perpendicular to the synoptic wind direction. The upwind edge of the region of lateral spread was found to be constrained by a topographic discontinuity such as a mountain ridge line. It was also observed that the active flaming zone often extended hundreds of metres downwind of the lateral spread region due to enhanced spotting. This rapid rate of lateral spread is at odds with the usual direction a fire would be expected to spread and the unexpected nature of this fire behaviour can result in significant danger to firefighters (Lahaye et al., 2017). This mode of fire spread has also been implicated in the development of violent pyro-convection (McRae et al., 2015).

Investigation of the phenomenon indicated that the atypical lateral spread was driven by a three-way interaction between synoptic winds, terrain and an active fire. Specifically, VLS was found to occur due to the tilting and stretching of ambient horizontal vorticity by the rising plume of a fire (hence the name). Research has also identified a number of environmental thresholds that need to be breached for VLS to occur. Sharples et al. (2012) initially expressed these thresholds in terms of a first-order wind-terrain filter, or binary variable $\chi$, which assumes a value of 1 in regions prone to VLS occurrence and 0 elsewhere.

While, the first-order filter identified the entire leeward slope as prone to VLS occurrence, subsequent laboratory experiments, wildfire observations and numerical simulations have revealed that the rapid lateral spread associated with VLS really only occurs in a relatively narrow portion of the leeward slope near the top of the hill (Quill and Sharples, 2015; Raposo et al., 2015; Simpson et al. 2016). This region could be better identified using a second-order VLS filter, based on the second-derivative of elevation, but for the idealised cases considered in this paper, a crude approximation will suffice. We therefore use a refined version of the first-order filter $\chi$ to define VLS prone regions. Specifically, we will consider parts of a landscape VLS-prone only if they are within 100 metres of the ridge line and have $\chi=1$. The grey and red shaded regions immediately downwind of the ridge lines that can be seen in Fig. 1, provide examples of the VLS-prone regions identified by the aforementioned second-order filter.

\section{NEAR-FIELD MODELLING OF VLS}

Hilton et al. (2018) detailed a two-dimensional fire spread model that uses a potential flow formulation to account for local air flows induced by the fire. The so-called 'pyrogenic potential' model simulates the pyrogenic air flow $\mathbf{u}_{p}$ close to the ground (mid-flame height). This pyrogenic flow can be added to the ambient air flow 
and the net wind field used to model the evolution of the fire. This model has been implemented as part of the Spark fire simulation framework (Miller et al., 2015), which is based on the level-set method.

The pyrogenic flow $\mathbf{u}_{p}$ is determined using a near-field approximation, which enables the use of Helmholtz decomposition:

$$
\mathbf{u}_{p}=\nabla \psi+\nabla \times \boldsymbol{\eta}
$$

for some scalar $\psi$ and some vector $\boldsymbol{\eta}$. Hilton et al. (2018) discuss how $\psi$ and $\boldsymbol{\eta}$ can be determined as solutions of the Poisson equations $\nabla^{2} \psi=\nu$ and $\nabla^{2} \boldsymbol{\eta}=\boldsymbol{\omega}$, where $\nu=-\partial_{z} u_{z}$ represents the derivative of the plume updraft, and $\boldsymbol{\omega}$ represents sources of vertical, $z$, vorticity. Once $\psi$ and $\boldsymbol{\eta}$ are known, the pyrogenic flow $\mathbf{u}_{p}$ can be determined to account for the effects of the fire on the local atmosphere.

a)

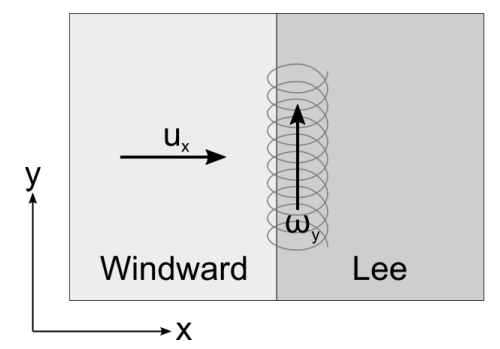

b)

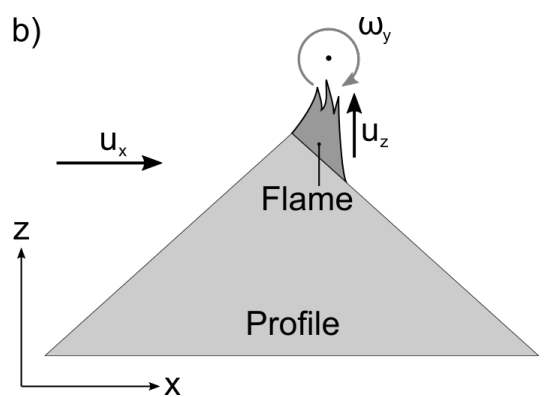

Figure 2. Schematic set-up of the modelling approach, a) top view and b) side view.

Sharples and Hilton (2019) considered the model set up as depicted in Fig. 2, and demonstrated how the solenoidal term in Eq. (1) can be set such that the near-field model emulates patterns of fire propagation consistent with VLS. In particular, it can be assumed that the separation of flow over the leeward slope creates horizontal vorticity $\left(\omega_{x}\right.$ and $\left.\omega_{y}\right)$, and that the vertical vorticity $\left(\omega_{z}\right)$ could be determined based on several assumptions for the flow dynamics on the leeward slope. Firstly, it can be assumed that the flow can be approximated as steady state as the outward spread of the fire is much slower than the wind flow. This means that the general steady-state inviscid vorticity equation can be used, given by (Vallis, 2017):

$$
(\mathbf{u} \cdot \nabla) \boldsymbol{\omega}=(\boldsymbol{\omega} \cdot \nabla) \mathbf{u}+\mathbf{s}
$$

where $\mathbf{u}$ is the flow field, $\boldsymbol{\omega}$ is the vorticity vector and $\mathbf{s}$ is a source term. Secondly, it can be assumed that the dominant flow in the vortex producing region is the vertical lofting flow created by the fire plume; that is, $u_{z} \gg u_{x}, u_{y}$. This allows for $u_{x}$ and $u_{y}$ components to be neglected in equation (2). Lastly, a linear scaling of vertical velocity with height in the vortex producing region was assumed, i.e. $\nu=-\partial_{z} u_{z}$, so that $u_{z}=\nu z$ (Hilton et al., 2018) with the standard no-flow boundary condition at ground level, $u_{z}=0$ at $z=0$.

Using the foregoing assumptions, Sharples and Hilton (2019) showed that the pyrogenic vertical vorticity due to a line source of ambient horizontal vorticity $\mathbf{s}_{x y}$ can be written as:

$$
\omega_{z}=k^{\prime} \delta\left(\left|\mathbf{x}-\mathbf{x}^{\prime}\right|\right) \delta(\phi)\left(\hat{\mathbf{n}} \cdot \mathbf{s}_{x y}\right)
$$

where $k^{\prime}$ is a constant based on factors including the vertical speed of the plume, the vortex strength, the nominal mid-flame height and $\nu$ (Sharples and Hilton, 2019). The function $\delta(x)$ is a Dirac delta function, the variable $\mathbf{x}^{\prime}$ is the nearest point on the line source to position $\mathbf{x}, \phi$ is the distance from the fire perimeter and $\hat{\mathbf{n}}$ is the outward normal of the fire perimeter. This gives rise to a source term where $\nabla u_{z} \cdot \mathbf{s}_{x y}>0$ and a sink where $\nabla u_{z} \cdot \mathbf{s}_{x y}<0$ resulting in two counter-rotating vortices, as illustrated in Fig. 3a. 
J.E. Hilton et al., Incorporating spotting in models of dynamic fire propagation

a)

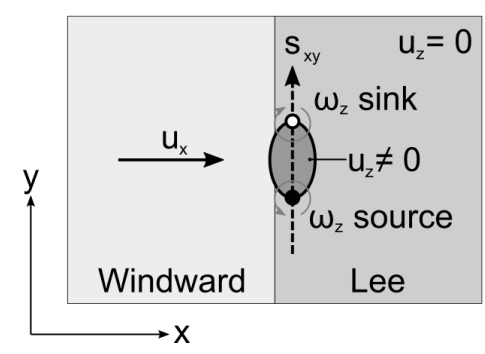

b)

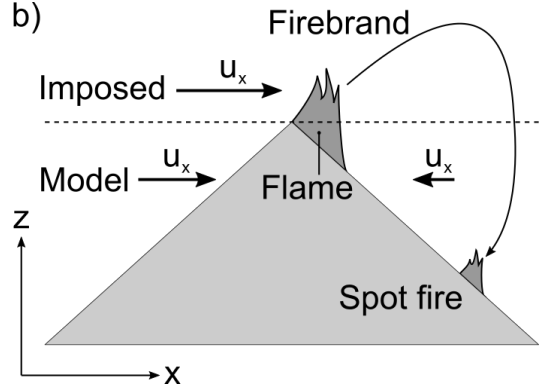

Figure 3. a) Resultant vorticity in the $z$ direction and circulation in the $x-y$ plane from an idealised plume. b) Schematic set-up of the firebrand modelling.

\section{INCORPORATING THE SPOTTING MODEL}

Firebrand transport and subsequent spot fire formation can clearly have a significant impact on fires under VLS conditions, as illustrated in Fig. 1. Firebrands were incorporated into the simulation using a Lagrangian particle model implemented in the Spark framework. The firebrands were assumed to be non-interacting and have negligible influence on the background wind. Additionally, any collision or coalescence of firebrands was not considered in this study. The motion of each firebrand was given by:

$$
m \frac{\partial \mathbf{v}}{\partial t}=\frac{1}{2} c_{D} A|\mathbf{u}-\mathbf{v}|(\mathbf{u}-\mathbf{v}) \mathbf{g}^{\prime}
$$

where $m$ is the mass of the firebrand, $\mathbf{v}$ is the firebrand velocity vector, $\rho$ is the air density $\left(\mathrm{kg} \mathrm{m}^{-3}\right), c_{D}$ the coefficient of drag for the firebrand and $A\left(\mathrm{~m}^{2}\right)$ is the cross-sectional area of the firebrand perpendicular to the flow. The reduced gravity vector $\mathbf{g}^{\prime}$ takes buoyancy effects into account and is given by $\mathbf{g}^{\prime}=[0,0,(b-1) g]$, where $g=9.8 \mathrm{~m} \mathrm{~s}^{-2}$ and $b$ is a dimensionless buoyancy parameter. The equation of motion for the firebrands, Eq. (4) was calculated using an adaptive 3rd order Bogacki-Shampine Runge-Kutta method.

Firebrands were randomly produced within the vorticity source region and launched at the slope angle, shown schematically in Fig. 3 b). The pyrogenic potential model results in a modified two-dimensional wind field, but firebrand transport requires a three-dimensional wind field. A straightforward method was used to account for the three-dimensional terrain effects in this study, where the flow acting on the firebrand was set to the imposed upwind flow speed above the terrain (above the dashed line in Fig. 3 b)) and set to the modelled speed below the terrain (below the dashed line in Fig. 3 (b)).

The dimensionless buoyancy parameter was used to model the lofting of the firebrands, but the correct range of values for this parameter in the context of real fires are currently an area of active research. This is a free parameter in this study, which we assume to range from 0 (firebrand is freely falling under gravity) to 1.5 (firebrand has a buoyancy 50\% the strength of gravity). Furthermore, the buoyancy must reduce as the plume cools and turbulent structures lofting the firebrand dissipate. Assuming a simple exponential cooling model, where buoyancy parameter reduces over time according to $b=b_{0} e^{-\kappa t}$, here $\kappa$ is a decay parameter.

\section{RESUlts}

Simulations of fire spread in the lee of a ridge using the models described in previous sections is shown in Fig. 4. The elevation is shown in grayscale and the colour scale represents the fire arrival time at each point. Ten minute isochrones are overlaid as solid black lines. The domain was a ridge $1 \mathrm{~km}$ high with a slope of $20^{\circ}$ on the windward side and $35^{\circ}$ on the lee slope. The ignition was initiated as a line $300 \mathrm{~m}$ in length and $50 \mathrm{~m}$ in width perpendicular to the ridge at a distance of $950 \mathrm{~m}$ from the ridge line. The simulation resolution was 10 $\mathrm{m}$ and the simulation was run for a period of 2 hours. The fire rate-of-spread, $R$, used the Rothermel equation (Rothermel, 1972) with a fuel moisture content of $8 \%$, a fuel load of 13.024 tonnes acre $^{-1}$ and a surface to volume ratio of $1159 \mathrm{ft}^{-1}$. Simulations took approximately 10 seconds on a K6000 GPU without firebrands and 110 seconds when firebrands were used.

The horizontal vorticity components ( $\omega_{x}$ and $\omega_{y}$ ) resulting from $\mathbf{s}_{x y}$ in Eq. (2) were not modelled in this study, 


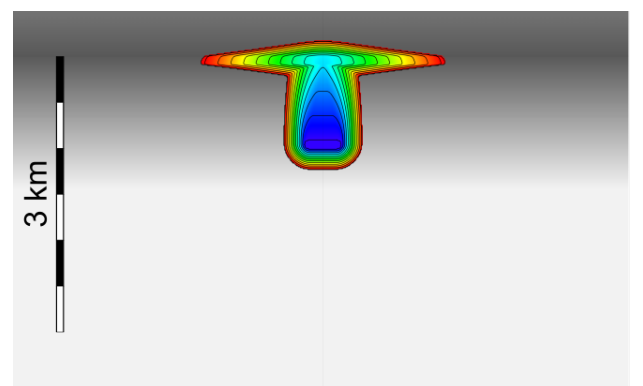

No firebrands

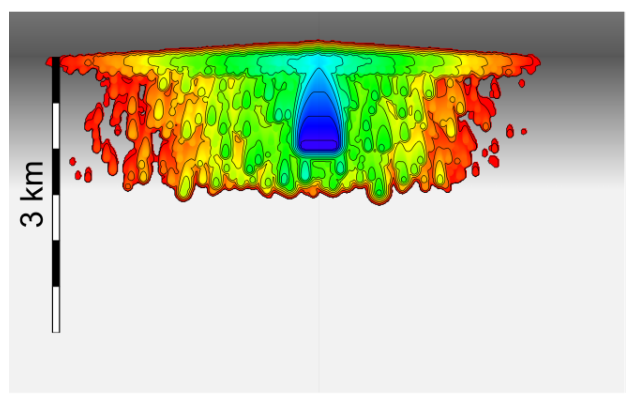

$\mathrm{K}=0.0050$

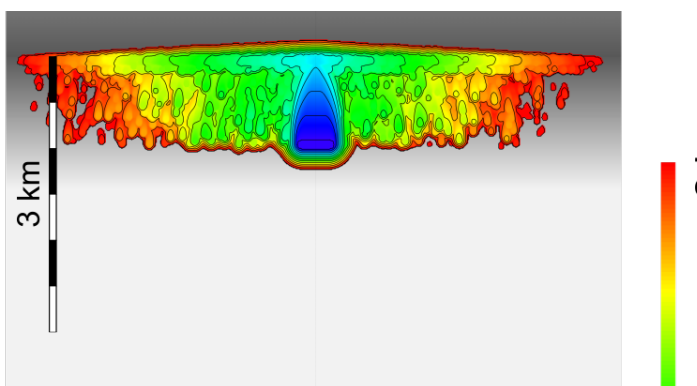

$\mathrm{K}=0.0075$

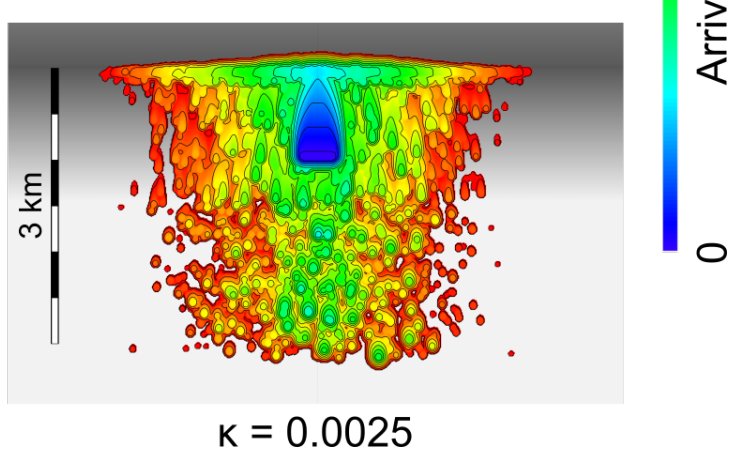

Figure 4. Lateral spread model with and without firebrands.

instead the recirculating wind flow on the lee of the hill was assumed to be static and steady state. The wind direction was set to be perpendicular to the ridge with a speed of $10 \mathrm{~m} \mathrm{~s}^{-1}$ on the windward slope and the re-circulation was prescribed by setting the wind speed to $-1 \mathrm{~m} \mathrm{~s}^{-1}$ on the lee slope. This imposed wind field was then modified by the pyrogenic potential model to account for vertical vorticity $\left(\omega_{z}\right)$ effects in the ground plane using Eq. (3). The vorticity was prescribed as a line source of $\mathbf{s}_{x y}=(0,1)$ through point $[-100,0]$, and $k^{\prime}=2000$. The position of the vortex line is consistent with the definition of VLS prone parts of the landscape discussed in the previous section.

Firebrands were uniformly produced within the vorticity source region at an average rate of 1 firebrand every 10 minutes per $10 \mathrm{~m} \times 10 \mathrm{~m}$ cell. Firebrands were created with a velocity vector of $\left[10,0,10 \tan \left(20^{\circ}\right)\right] \mathrm{m} \mathrm{s}^{-1}$. All firebrands were modelled as spheres of radius $5 \mathrm{~mm}$ and density $250 \mathrm{~kg} \mathrm{~m}^{-3}$. The mass loss of the firebrands was not modelled, although this may be important for long range firebrands (Tarifa et al., 1965). The values of $b_{0}$ were chosen from a uniform random distribution of $[1,1.5]$ and a range of $\kappa$ values were tested, $\kappa=0.0075,0.005,0.0025$. For comparison, a simulation is also shown in Fig. 4 with no firebrand transport or spot fire formation.

\section{Discussion ANd Conclusions}

The combined VLS and firebrand models appear to qualitatively replicate the fire behaviour shown in Fig. 1 despite the range of simplifications used for the models. There are, however, a number of assumptions and unknowns in the model which require calibration and further research. These include the vortex strength parameter $k$, the firebrand rate of production and the plume decay constants $b_{0}$ and $\kappa$. Despite this, the simulations indicate a strong dependency between the lofting buoyancy and the vorticity driven effects. Small values of the decay parameter, representing longer cooling times of the plume and longer lofting, resulted in mass spot fire formations downwind of the ridge apparently similar to 'deep flaming' events. Larger values of the decay parameter, representing short plume cooling times and shorter lofting, resulted in an enhanced lateral spread. This appeared to be due to firebrands falling close to the vortices at the edge of the lateral spread region, thereafter being entrained and subsequently starting new spot fires at the end of the region.

We have demonstrated that these models can be implemented in a fire spread simulator, Spark, and can be run at speeds greatly exceeding real-time suitable for operation use. Further work must be carried out to examine the range of validity of the models and include further physical effects such as fully resolved horizontal re- 
circulation and firebrand combustion. However, simulations using these models could provide information on counter-intuitive modes of fire behaviour for management and risk assessment. Future studies will investigate the applicability of the models to more complex scenarios and compare the results of the model to real-world data.

\section{ACKNOWLEDGEMENT}

The authors acknowledge the support of the Bushfire and Natural Hazards Cooperative Research Centre. Part of this work was also performed in the framework of Project Firewhirl, with reference PTDC/EMS321/ENE/2530/2014, supported by the Portuguese Foundation for Science and Technology, with National Funds. The authors are also grateful to the the NSW Rural Fire Service for provision of line scan imagery.

\section{REFERENCES}

Finney, M. A. (2004). Farsite: Fire area simulator: model development and evaluation. Research paper RMRS-RP-4 Revised, USDA Forest Service, Rocky Mountain Research Station.

Hilton, J., A. Sullivan, W. Swedosh, J. Sharples, and C. Thomas (2018). Incorporating convective feedback in wildfire simulations using pyrogenic potential. Environmental Modelling \& Software 107, 12-24.

Lahaye, S., J. Sharples, S. Matthews, S. Heemstra, and O. Price (2017). What are the safety implications of dynamic fire behaviours? In G. Syme, D. Hatton MacDonald, B. Fulton, and J. Piantadosi (Eds.), MODSIM2017, 22nd International Congress on Modelling and Simulation, pp. 1125-1130. Modelling and Simulation Society of Australia and New Zealand.

McRae, R. (2004). The breath of the dragon - observations of the January 2003 ACT bushfires. In Proceedings of Bushfire 2004 - Earth, wind \& fire: fusing the elements, 25-28 May, Adelaide.

McRae, R., J. Sharples, and M. Fromm (2015). Linking local wildfire dynamics to pyroCb development. Natural Hazards and Earth System Sciences 15(3), 417-428.

Miller, C., J. Hilton, A. Sullivan, and M. Prakash (2015). Spark-a bushfire spread prediction tool. In R. Denzer, R. Argent, G. Schimak, and J. Hřebíček (Eds.), Environmental Software Systems. Infrastructures, Services and Applications. Vol. 448 of IFIP Advances in Information and Communication Technology, pp. 262-271. Springer International Publishing.

Quill, R. and J. Sharples (2015). Dynamic development of the 2013 aberfeldy fire. In T. Weber, M. McPhee, and R. Anderssen (Eds.), MODSIM2015, 21st International Congress on Modelling and Simulation, pp. 284-290. Modelling and Simulation Society of Australia and New Zealand.

Raposo, J., S. Cabiddu, D. Viegas, M. Salis, and J. Sharples (2015). Experimental analysis of fire spread across a two-dimensional ridge under wind conditions. International Journal of Wildland Fire 24(7), 1008-1022.

Rothermel, R. (1972). A mathematical model for predicting fire spread in wildland fuels. Research Paper INT-115, USDA Forest Service, Intermountain Forest and Range Experiment Station.

Sharples, J. and J. Hilton (2019). Modelling vorticity-driven wildfire behaviour using near-field techniques. Frontiers in Mechanical Engineering (under review).

Sharples, J., R. McRae, and S. Wilkes (2012). Wind-terrain effects on the propagation of wildfires in rugged terrain: fire channelling. International Journal of Wildland Fire 21(3), 282-296.

Simpson, C., J. Sharples, and J. Evans (2014). Resolving vorticity-driven lateral fire spread using the WRF-fire coupled atmosphere-fire numerical model. Natural Hazards and Earth System Sciences 14(9), 2359-2371.

Simpson, C., J. Sharples, and J. Evans (2016). Sensitivity of atypical lateral fire spread to wind and slope. Geophysical Research Letters 43(4), 1744-1751.

Simpson, C., J. Sharples, J. Evans, and M. McCabe (2013). Large eddy simulation of atypical wildland fire spread on leeward slopes. International Journal of Wildland Fire 22(5), 599-614.

Tarifa, C. S., P. P. del Notario, and F. G. Moreno (1965). On the flight paths and lifetimes of burning particles of wood. Symposium (International) on Combustion 10(1), 1021 - 1037. Tenth Symposium (International) on Combustion.

Tolhurst, K., B. Shields, and D. Chong (2008). Phoenix: development and application of a bushfire risk management tool. Australian Journal of Emergency Management 23(4), 47.

Vallis, G. K. (2017). Atmospheric and oceanic fluid dynamics. Cambridge University Press.

Viegas, D. X. (2006). Parametric study of an eruptive fire behaviour model. International Journal of Wildland Fire 15(2), 169-177.

Viegas, D. X. and L. P. Pita (2004). Fire spread in canyons. International Journal of Wildland Fire 13(3), 253-274. 